\title{
Verification and Diagnosis of Digital Systems by Ternary Reasoning
}

\author{
Ayman M. Wahba and Einar J. Aas \\ Joseph Fourier University, IMAG-ARTEMIS Laboratory, Grenoble 38041, FRANCE
}

\begin{abstract}
Ternary vectors $\{0,1, X]$ may be used to simulate binary systems more efficiently than binary vectors. It has recently been shown by R.E. Bryant that formal verification by ternary simulation is feasible. In this paper, we demonstrate that complete verification of Finite State Machines is possible by ternary simulation. The verifica ion vectors are derived from AND/OR trees. We also show how design error diagnosis can be performed by utilizing the difference vector set. Algorithms for the diagnosis of single inverter errors, and wrong gate type, are presented, together with illustrative examples.
\end{abstract}

\section{Introduction}

Formal methods for design verification of digital systems have been investigated for many years. The past 5 years has seen lots of research activity in formal verification, and practical methods are beginning to appear. An overview of methods can be found in [GUP92], and utilization of VHDL as a design description instrument for formal verification is presented in [BOR92]. Numerous other papers on formal verification exist, but few papers have been concerned with the more difficult problem of design error diagnosis, including error correction, when an error is proven to exist. Some researchers have addressed this p.oblem, notably [MAD89], [TAM89], [TOM90] and [KUO92]. A typical approach to verification of digital systems is to transform a specification description (SPEC) and an implementation description (IMPL) to a common canonical basis where the ' may be compared directly.

The most widely used basis is that of Ordered Binary Decision Diagrams (OBDDs) [BRY86],[MAD89], but other methods are also in use, (see [GUP92]). Symbolic simulation may be ut ized for verification, e.g. to extract Boolean expressions to be verified afterwar is by OBDDs. This technique is used in [CLA91] for verification of signal flow subg:aphs.

Despite the fact that modern logic simulators offer at least a ternary set of signal values, e.g. $v=\{0,1, X\}$, where $X$ represents "unknown" or "don't care", this verification opportunity has normally not been exploited systematically for design verification. Simulation as a verific ation tool has recently gained attention. R. Bryant [BRY91a] established theoretical fc undations for formal verification by simulation, in particular 3-value simulation. Of importance is the concept of partial ordering $(X<0, X<1)$ based upon information content. One input vector $V$ is weaker than another vector $\mathrm{V}$ ' if $\mathrm{V}$ can be created by assigning " $\mathrm{X}$ " to one or more bits of value " 0 " or " 1 " in $\mathrm{V}$ '. If a response is valid for a vector $\mathrm{V}$ containing ' $k$ ' $\mathrm{X}$ 's, then it is valid for all $2^{k}$ binary vectors stronger than $V$. We will utilize AND/OR trees [HWA85] for ternary vector extraction, and as $\mathrm{z}$ basis for reasoning about diagnosis

The diagnosis problem is indeed of great practical importance buth for manually designed and/or optimized circuits, as well as for bug tracking in automatic synthesis programs. In particular, repeated design changes to satisfy, e.g. speed requirements, are often done manually, and the need for efficient diagnosis is particularly pressing. 
Publications on diagnosis of design errors are few. [MAD89] presented an automated diagnosis and rectification system for debugging of gate level descriptions, based upon equation solving facilities of the theorem prover. [TOM90] uses an inventive technique of finding patterns with only one Boolean variable $\mathrm{X} / \mathrm{X}^{\prime}$ used to sensitize design errors. A special logic simulator allowing this variable is needed. [KUO92] utilizes test pattern generation techniques to provoke and localize design errors.

We focus on verification and diagnosis of combinational circuits and Mealy/Moore type Finite State Machines (FSMs), with given input, output and state assignments in this paper. This is not considered as a limitation, because in practise the assignments are known by the designer (or synthesis system).

This paper is organized as follows: Section 2 discusses similarities and differences between design error and production error diagnosis, Section 3 introduces notation and definitions and a brief recollection of the results of Bryant's proof of 3-value simulation as a formal verification method. In Section 4, we formulate the verification problem, introduce the AND/OR trees, and offer an example of a ternary set for complete verification. In Section 5 we formulate the diagnosis problem by introducing the difference function, and in Section 6 we propose a diagnostic procedure based upon error hypotheses related to practical experience of design errors. Section 7 and 8 offer examples of error hypotheses, and theorems for tree structured circuits to be used as basis for diagnostic procedures for more general circuits are presented together with simple examples. Section 9 yields conclusions and further work.

\section{Can We Import Methods from Testing?}

Conceptually, each design error has an error location. For example, the "missing/extra inverter" error is localized to a single lead, while "wrong gate type" (e.g. AND instead of OR) is located to a gate (or to the gate output). It is convenient to consider the signal immediately suffering from a design error as the error location L. Let us consider single design error locations first. Two concepts may conveniently be imported from the area of testing (see e.g. [SEL68]): Error excitation (also called error control), and error sensitization (aiso called error observation). Error excitation $E(L)$ is the condition for exposing the error, i.e. yielding the opposite value on $L$ in presence of the design error. Error sensitization $S(L, Y)$ is the condition for propagating the effect of the error to an output $Y$. Formally, the necessary and sufficient conditions for detecting a design error at $\mathrm{L}$ by observing the output $\mathrm{Y}$ may be expressed as:

$$
\operatorname{DET}(\mathrm{L}, \mathrm{Y})=[\mathrm{L}(\mathrm{V}) \oplus \mathrm{LC}(\mathrm{V})] \cap \mathrm{S}(\mathrm{L}, \mathrm{Y})
$$

where $\mathrm{L}=\mathrm{L}(\mathrm{V})$ is a Boolean function of the input vector $\mathrm{V}$, and $\mathrm{LC}(\mathrm{V})$ is the correct value on lead $\mathrm{L}$. The Boolean difference [SEL68] is:

$$
\mathrm{S}(\mathrm{L}, \mathrm{Y})=\mathrm{dY} / \mathrm{d} \mathrm{L}=\mathrm{Y}(\mathrm{L}=0) \oplus \mathrm{Y}(\mathrm{L}=1),
$$

The corresponding techniques of localizing a manufacturing defect after testing, however, may not be used direct.y for design error diagnosis, for the following reasons: 
- Test programs will be run on real hardware, which only can generate binary, not ternary values. Thus, no complete test programs can be made for VLSI circuits.

- Test programs are consequently made to generate $100 \%$ fault coverage for a limited set of fault models, e.g. the universally used stuck-at faults.

- Fault dictionaries may be built for diagnosis. These dictionaries provide a fault tree based upon the faulty response of each modelled fault. But exact match is not found when there is a non modelled fault in the circuit, and thus probing of signals in the known circuit structure is often used to localize the fault.

- For diagnosis of design errors, we do not know the correct circuit structure, but only the correct behavior.

- We may take advantage of ternary simulation or reasoning for locating design errors.

However, it is impossible to use (1) to generate a test for each conceivable design error, because the number of possible design errors is unlimited.

Instead, we want to build our diagnosis upon the computed difference set, and upon reasoning about signals and gates in the given structure. We start with simplifying assumptions in order to find reasonable heuristics that work well for commonly encountered design errors, and add more heuristics as needed. It is also necessary to interact with the designer in order to get approval for partial design fixes.

\section{Notation and Definitions}

An $n$-bit (input or output) vector is written $Z=<z_{1} z_{2} \ldots z_{n}>$, and may be binary $\left(B^{n}\right)$ or ternary $\left(\mathrm{T}^{\mathrm{n}}\right)$. We define:

$$
\begin{aligned}
& \text { B: }\{0,1\} \text { - the Boolean domain. } \\
& T:\{0,1, X\} \text { - the ternary domain, where } X \in\{0,1\} . \\
& U^{n}:\{0,1\}^{n} \text { - the universal set, denoted }<X X \ldots X>\text {. }
\end{aligned}
$$

A Boolean function $\mathrm{Y}$ from $\{0,1\}^{\mathrm{n}}$ to $\{0,1\}$ may be completely or incompletely specified over $\{0,1\}^{n}$. An incompletely specified function carries a CARE-set: $C \in$ $\{0,1\}^{\mathrm{n}}$. The CARE-set includes the ON-set $\mathrm{C}_{1}$ and the OFF-set $\mathrm{C}_{0}: \mathrm{C}=\mathrm{C}_{0} \cup \mathrm{C}_{1}$, and the function is uniquely specified by the pair $(C, Y)$.

\subsection{A Simulator Model}

For the benefit of theorems to be proven, we define the functional properties of a simple, generic 3-value logic simulator GEN_3V_SIM. Most, if not all, commercial simulators obey these properties on a subset of their value alphabet. We consider the monotonic gate types (AND,OR,NOT,NAND,NOR). An example of a gate function is:

$$
\mathrm{AND}=\{0 \text { if one input is } 0,1 \text { if all inputs are } 1, \mathrm{X} \text { otherwise }\} .
$$

The circuit model is simply a Directed Acyclic Graph (DAG) of interconnected gates. The simulation model of the circuit can be considered as a function O(IMPL,I), which monotonically maps an input vector $I$ into a response vector $O$ of IMPL in the ternary domain: $T^{\mathrm{n}} \rightarrow \mathrm{T}^{\mathrm{m}}$. 


\subsection{The Formal Foundation}

[BRY91a] introduced a partial ordering $X<0, X<1$ based upon "information content". This ordering may be extended to vectors. Given $V, V^{\prime} \in T^{n}$, then $\mathrm{V}<\mathrm{V}^{\prime}$ (less than, or weaker than) if each element of $V$ is less than or equal to the corresponding element of $\mathrm{V}^{\prime}$, and at least one element of $\mathrm{V}$ is weaker than the corresponding element of $\mathrm{V}^{\prime}$. Examples are:

$$
<01 \mathrm{XX}><<011 \mathrm{X}>\text {, while }<01 \mathrm{XX}>\text { and }<10 \mathrm{XX}>\text { are inconsistent. }
$$

The usefulness of this ordering concept becomes evident when we investigate our model of the logic simulator. The basic gate types defined are monotonic functions, for which the following monotonicity property is satisfied:

$$
\mathrm{V} \leq \mathrm{V}^{\prime} \Rightarrow \mathrm{g}(\mathrm{V}) \leq \mathrm{g}\left(\mathrm{V}^{\prime}\right) \Rightarrow \mathrm{O}(\text { IMPL, } \mathrm{V}) \leq \mathrm{O}\left(\text { IMPL, } \mathrm{V}^{\prime}\right) \text {. }
$$

Where the last monotonicity property is valid for the state values and output values for the simulator model, when each gate function is a monotonic function. In (other) words: a valid response $O$ of IMPL to an input vector $V$ is also a valid response to every stronger vector $\mathrm{V}^{\prime}>\mathrm{V}$.

\section{A Formulation of the Verification Problem}

\subsection{Necessary and Sufficient Conditions for a Ternary Input Vector Set}

Bryant exploited an assertion method for FSMs similar to the Floyd-Hoare assertion method in program verification [BRY91a]. In our case, we are considering an FSM with complete control/observation of the states, and thus it is not necessary to distinguish between PIs and SIs (Primary and Secondary Inputs), or between POs and SOs (Primary and Secondary Outputs). We are searching for a complete set of assertions (pairs of predicates), which in this case looks like: [INPUT] RESPONSE.

These assertions may be retrieved automatically from the specification (SPEC) of the circuit, or we may also retrieve these assertions from the proposed IMPL. In any case, the set of INPUT vectors must cover the CARE-set to be complete, and the response must belong to $\{0,1\}$.

The immediate question that arises is of course: How do we derive a validating vector set? Before we present our method of deriving a validating vector set $\mathrm{V}$, let us illustrate the problem at hand by a simple example, see Fig. 1.

\begin{tabular}{|llll|l|}
\hline $\mathrm{A}$ & $\mathrm{B}$ & $\mathrm{C}$ & $\mathrm{D}$ & $\mathrm{Y}$ \\
\hline $\mathrm{X}$ & $\mathrm{X}$ & 1 & 0 & 1 \\
1 & 0 & $\mathrm{X}$ & $\mathrm{X}$ & 1 \\
$\mathrm{X}$ & 0 & $\mathrm{X}$ & 0 & 1 \\
\hline $\mathrm{X}$ & 1 & 0 & $\mathrm{X}$ & 0 \\
0 & $\mathrm{X}$ & $\mathrm{X}$ & 1 & 0 \\
\hline
\end{tabular}

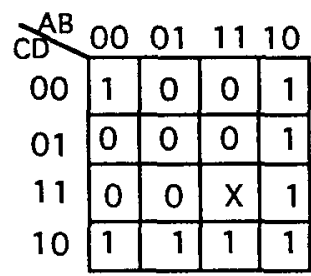

Fig.1. Specification and Karnaugh map illustration of a function Y(A,B,C,D). 
The specification is given as a CARE-set in the ternary notation (also called cubes), and the Karnaugh map is included for illustration. Any ON-set, or OFF-set that covers this specification, may be a candidate set for verification.

\subsection{Utilization of AND/OR Trees for Derivation of Ternary Vectors}

From a proposed IMPL, we may create AND/OR trees of the circuit. A strategy for verification of a proposed IMPL is thus: "Create complete ON- and OFF-sets for IMPL, and compare these against SPEC for verification". The comparison will be explained in Sec. 5.2. We will introduce the AND/OR tree by way of an example below.

Example 1. We propose an implementation of the SPEC given in Fig. 1 based upon the Boolean equation: $\mathrm{W}=\mathrm{AB}^{\prime}+\left(\mathrm{D}+\left(\mathrm{C}+\mathrm{B}^{\prime}\right)^{\prime}\right)^{\prime}$. An erroneous circuit diagram is given in Fig. 2 (f should be inverted), together with the ternary vector set derived from the AND/OR trees. Fig. 3 shows the corresponding AND/OR trees of the circuit, from which the ternary ON/OFF sets given, are derived. The verification is done by computing the difference set to be defined in (5), and checking if it is empty. (It is not). End Example 1.

The AND/OR tree for the ON-set is created as follows: Start from the output W, and find the conditions on the inputs $E, G$ for $W$ to be "1". These are obviously: $(E=1$ OR $\mathrm{G}=1$ ). Thus, two nodes labeled $\mathrm{E}$ and $\mathrm{G}$ are created, and connected to the $\mathrm{W}$ node by OR branches. Furthermore, $G=1$ requires $(D=0$ AND $F=0$ ). Thus, two nodes labeled $D$ and $F$ are created, and connected to the G node by AND branches. AND is indicated with an arch, no arch indicates $O R$. The " 0 " condition is shown by $\mathrm{D}^{\prime}, \mathrm{F}^{\prime}$. Inverted signals are not labeled explicitly. Note that fanouts present constraints to the lead values. For example: $\mathrm{B}_{1}=\mathrm{B}_{2}$. The $\mathrm{AND} / \mathrm{OR}$ tree for the OFF-set is created by requiring $\mathrm{W}=0$. It is easy to show that the corresponding tree can be created directly from the ON-tree by inverting every node, and exchanging AND $\leftrightarrow$ OR.
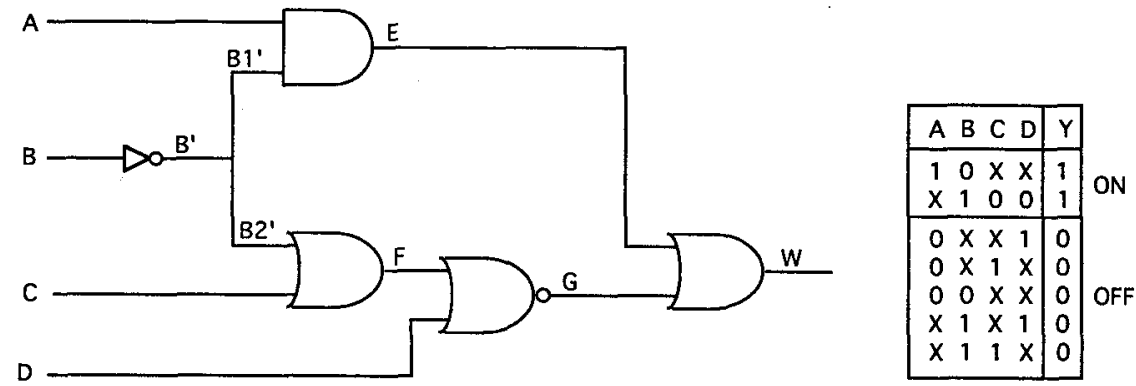

Fig. 2. Circuit diagram and ternary vector set for Ex. 2.

\section{A Formulation of the Diagnosis Problem}

The problem of design error diagnosis (see fig. 4) may be formulated as follows:

"Given a specification SPEC and a implementation candidate IMPL of a circuit. If it is proven that IMPL is not functionally correct (i.e. design error detection), then propose design changes, and prove that these will yield a functionally correct circuit". Notice that diagnosis includes rectification and reverification. 

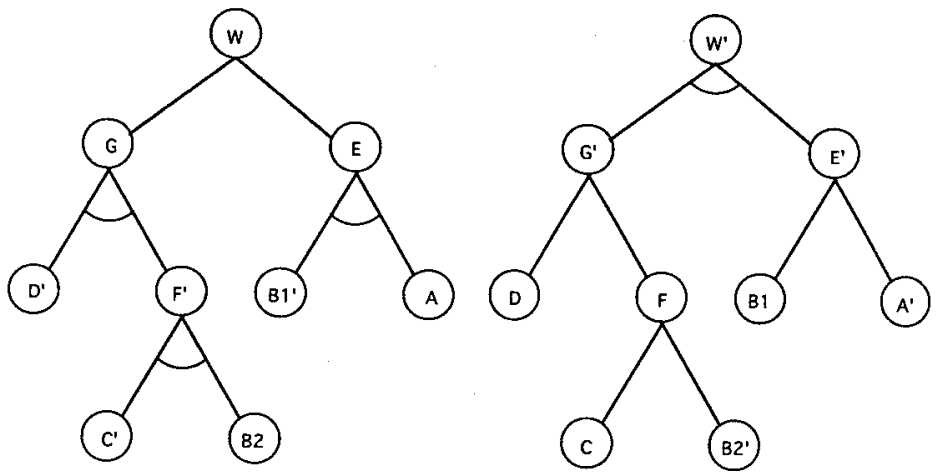

Fig. 3. ON and OFF AND/OR trees for Example 1, and the derived ON/OFF-sets.

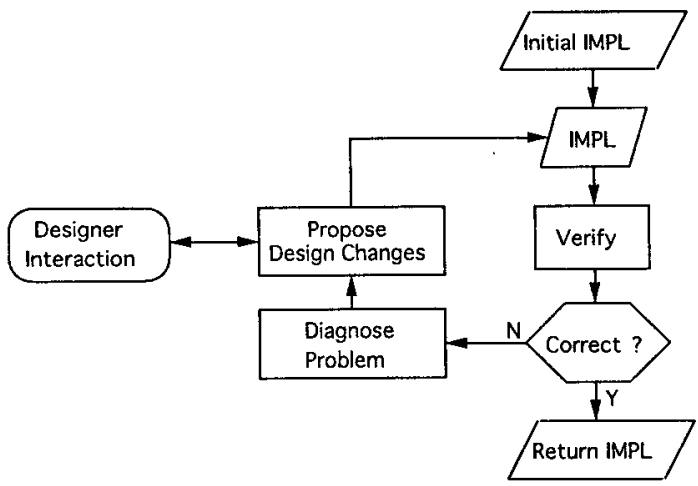

Fig. 4. The diagnosis procedure.

\subsection{A Single Solution to Diagnosis is not Available}

There is no single solution to the diagnosis problem, which may be easily proven by the following argument (restricted to single output, fully specified functions for notational convenience): Given a specified function $\mathrm{Y}$, and an implemented function W. We define the difference function in the Boolean domain by utilizing the $\mathrm{XOR}(\operatorname{ADD}(\bmod 2))$ operator:

$$
\mathrm{Q}=\mathrm{Y} \oplus \mathrm{W}
$$

A correction of IMPL is achieved by adding $(\bmod 2) \mathrm{Q}$ to the function $\mathrm{W}$ to obtain a new function $\mathrm{CY}$ :

$$
\mathrm{CY}=\mathrm{Q} \oplus \mathrm{W}
$$

$C Y$ is correct by construction: $C Y=Q \oplus W=Y \oplus W \oplus W=Y \oplus 0=Y$. We may now transform CY to infinitely many functionally correct circuits by applying legal Boolean transformation rules. But the design, seen as a structure of gates and interconnections, is almost correct, i.e. a few (hopefully one) changes will produce a correct circuit. Thus, a pragmatic approach to diagnosis may be stated as follows: 
"Given an incorrect candidate implementation IMPL of SPEC, propose as few design changes as possible that will produce a correct IMPL with respect to SPEC".

\subsection{The Difference Set}

A proposed IMPL will have an ON-set $\mathrm{W}_{1}$, and an OFF-set $\mathrm{W}_{0}$. Correspondingly, we may compute the $O N$-set $Y_{1}$, and the OFF-set $Y_{0}$ from the given SPEC. We define the low and high difference sets, and the total difference set thus:

$$
D_{0}=\left\{Y_{1} \cap W_{0}\right\}, D_{1}=\left\{Y_{0} \cap W_{1}\right\}, D(Y, W)=\left\{D_{0}, D_{1}\right\}
$$

Note that a ternary vector belongs to $D_{0}\left(D_{1}\right)$ if it yields either $Y=1(Y=0)$ or $Y=X$. We distinguish between these responses, therefore we divide $D_{0}$ and $D_{1}$ into:

$$
D_{0}=\left\{D_{01}, D_{0 X}\right\}, D_{1}=\left\{D_{10}, D_{1 X}\right\},
$$

where $D_{0 X}\left(D_{1 X}\right)$ is the set leading to $Y=X$. This normally indicates that the implicant covers some minterms in $Y_{0}$, and some minterms in $Y_{1}$. Before initiating diagnosis, we compute these difference sets from the AND/OR trees of IMPL, and the given SPEC. A preanalysis of $D(Y, W)$ for each output provide diagnostic information:

- The design error must reside in the area reached by backtracing from outputs where $\mathrm{D}(\mathrm{Y}, \mathrm{W})$ is non EMPTY. Such backtracing may limit the search area.

- If $\mathrm{D}=\left\{\mathrm{Y}_{0}, \mathrm{Y}_{1}\right\}$, then the error is "inverted output $\mathrm{Y}$ ".

- If either $\mathrm{D}_{01}=\mathrm{EMPTY}$, or $\mathrm{D}_{10}=\mathrm{EMPTY}$, the design error has changed $\mathrm{Y}$ monotonically, and we can restrict ourselves to monotonic errors only.

\section{Diagnosis by Error Hypotheses}

Knowledge about typical design errors. will aid the reasoning process. We propose a method based upon "error hypotheses": Based upon experience, a set of error classes is hypothesised, and a diagnosis procedure is followed for each class. The error hypotheses are tested in order of probability of occurrence. For illustration, we refer to [AAS92], where design error statistics is collected. The design error classification, and the number of occurrences of each error, are reproduced in Tab. II.

\begin{tabular}{|l|c|c|}
\hline DESIGN ERROR CLASS & NUMBER & $\%$ \\
\hline Missing/extra inverter & 91 & $48 \%$ \\
Wrong gate type & 35 & $19 \%$ \\
Incorrectly placed input wire & 32 & $17 \%$ \\
Missing gate input (missing fanout) & 17 & $9 \%$ \\
Missing gate & 9 & $5 \%$ \\
Other & 4 & $2 \%$ \\
\hline
\end{tabular}

Table II. Design error classes, and frequency of occurrence

Note the frequency of inverter errors. By "missing/extra inverter" error, we mean a signal lead with wrong polarity. Interestingly, only 5 classes are needed to capture $98 \%$ of the design errors. 
A decision tree may be built to guide diagnosis, see Fig. 5. The decision boxes indicate diagnosis hypotheses (e.g. STR_INV is Strategy Inverter Error), and the tree is traversed depth-first until a design error hypothesis is proven true. The leaf nodes indicate strategies or procedures to be invoked for each error hypothesis.

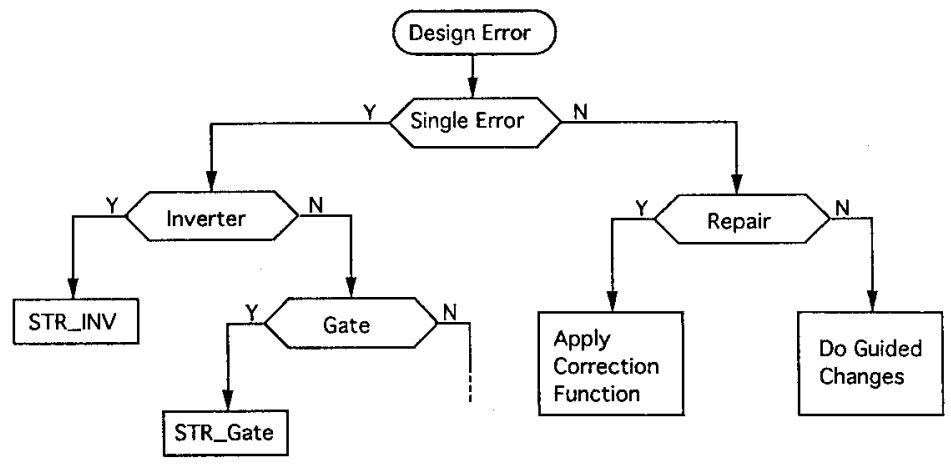

Fig. 5. Decision tree for diagnosis strategy.

We believe that the AND/OR tree, which is an isomorphic mapping of the circuit structure, with each gate and lead of the circuit uniquely identified, is very well suited for diagnosis, because plausible error hypotheses may be tested easily by modest changes to the AND/OR tree, followed by implications. In the next two sections, we will develop strategies for two classes of design errors.

\section{Exact Diagnosis of Single Inverter Errors}

The SINGLE_INVERTER error hypothesis postulates that by inverting a single lead in the circuit, the faulty circuit will be converted to a correct circuit. We show how diagnosis may be obtained by exploiting the AND/OR graphs. We use graphs with one unique node for each fanout branch in the case of diagnosis. Fig. 3 exhibits two such nodes, $\mathrm{B}_{1}{ }^{\prime}$ and $\mathrm{B}_{2}$ ', as fanouts from $\mathrm{B}^{\prime}$. Of course, $\mathrm{B}_{1}=\mathrm{B}_{2}=\mathrm{B}$ for the actual IMPL, but the search for design errors may lead us to alter one or more given implications.

Our goal is to find a way to exactly locate the inverter error by maximum utilization of the difference set. We check a postulated design error hypothesis by incurring a candidate correction, and deriving its implication (by e.g. ternary simulation). First, we demonstrate how inverter errors may be located in a tree structured circuit. We will prese at a theorem on which an algorithm may be constructed.

Definition: The Cone of Influence of a lead L, COI(L), is the set of every input that exhibits a path to $\mathrm{L}$.

Theorem 1. Given a tree structured circuit IMPL obeying the SINGLE_INVERTER error hypothesis, an error location L, the Cone of Influence $\operatorname{COI}(\mathrm{L})$, and the ternary difference vector set $\mathrm{D}(\mathrm{Y}, \mathrm{W})=\left\{\mathrm{D}_{0}, \mathrm{D}_{1}\right\}$, where $\mathrm{D}_{0}$ belongs to W's OFF-set, and $\mathrm{D}_{1}$ belongs to W's ON-set. For every input $\mathrm{X}_{\mathrm{i}} \in$ $\operatorname{COI}(L)$, there will be at least one vector $V_{j} \in D_{0}$ with $X_{i}=v$, and one vector $V_{k} \in$ $D_{1}$ with $X_{i}=v '$. End Theorer 1. 
Proof. For any $X_{i} \in \operatorname{COI}(L)$ it is possible to find one vector $V$ which yields $\mathrm{dW} / \mathrm{dX} \mathrm{X}_{\mathrm{i}}=1$. This yields a unique sensitive path from $\mathrm{X}_{\mathrm{i}}$ to $\mathrm{W}$ through $\mathrm{L}$. By inverting $X_{i}$ from $V$ to $V^{\prime}$ one obtains another input vector $V N$, and all signal values on the path will be inverted. Furthermore, both $\mathrm{V}$ and $\mathrm{VN}$ belong to the difference set $\mathrm{D}(\mathrm{Y}, \mathrm{W})$, one in each partition $\left\{\mathrm{D}_{0}, \mathrm{D}_{1}\right\}$. Q.E.D.

Corollary 1 . Only $X_{i} \in \mathrm{COI}(\mathrm{L})$ obeys the property of theorem 2 .

Theorem 1 and its corollary may be utilized to develop an algorithm for finding the exact error location under the SINGLE_INVERTER error hypothesis By theorem 1, we realize that we should be looking for pairs of vectors in the $\left\{D_{0}, D_{1}\right\}$ sets that differs in just one position. By collecting these input positions, we obtain the Cone of Influence for $\mathrm{L}$, from which $\mathrm{L}$ is uniquely determined. The algorithm is given below.

\section{Algorithm FIND_INV_LOCATION:}

1. Compute the two partitions $\left\{\mathrm{D}_{0}, \mathrm{D}_{1}\right\}$ of the difference set.

2. Perform bit-by-bit XOR of every vector in $D_{0}$ with every vector in $D_{1}$. Result: $\mathrm{D}_{01}$.

3. Perform bit-by-bit $O R$ of all vectors in $D_{01}$. Result: $V_{01}$.

4. $\mathrm{COI}(\mathrm{L})=$ set of $X_{i}=1$ in $V_{01}$.

5. Find $\mathrm{L}$ by propagating $\mathrm{COI}(\mathrm{L})$ to common point of convergence. End Algorithm FIND_INV_LOCATION.

This method also serves as a heuristic to locate errors in circuits with fanouts. We will consider each fanout branch as a pseudo-input, and use the algorithm FIND_INV_LOCATION also for non-tree structured circuits. The error location(s) produced by the algorithm must be verified by error injection and implication. This is conveniently done in our model. According to (4), the new circuit is correct if its difference set covers the difference set $D(Y, W)$. We will illustrate the use of ALGORITHM FIND_INV_LOCATION by a small example below.

\begin{tabular}{|c|c|c|c|c|}
\hline A & B & C & D & W \\
\hline 1 & 0 & $x$ & $\mathrm{X}$ & 1 \\
\hline $\mathrm{X}$ & 1 & 0 & 0 & 1 \\
\hline 0 & $X$ & $\mathrm{X}$ & $\frac{1}{x}$ & 0 \\
\hline 0 & $X$ & 1 & $\mathrm{X}$ & 0 \\
\hline 0 & 0 & $X$ & $X$ & 0 \\
\hline$X$ & 1 & $X$ & 1 & 0 \\
\hline$X$ & 1 & 1 & $X$ & 0 \\
\hline
\end{tabular}

\begin{tabular}{|r|r|r|r|r|r|}
\hline$A$ & $\mathrm{BI}$ & $\mathrm{B} 2$ & $\mathrm{C}$ & $\mathrm{D}$ & $W$ \\
\hline 1 & 0 & $\mathrm{X}$ & $\mathrm{X}$ & $\mathrm{X}$ & 1 \\
$\mathrm{X}$ & $\mathrm{X}$ & 1 & 0 & 0 & 1 \\
0 & $\mathrm{X}$ & $\mathrm{X}$ & $\mathrm{X}$ & 1 & 0 \\
0 & $\mathrm{X}$ & $\mathrm{X}$ & $\mathrm{J}$ & $\mathrm{X}$ & 0 \\
0 & $\mathrm{X}$ & 0 & $\mathrm{X}$ & $\mathrm{X}$ & 0 \\
$\mathrm{X}$ & 1 & $\mathrm{X}$ & $\mathrm{X}$ & 1 & 0 \\
$\mathrm{X}$ & $\mathrm{I}$ & $\mathrm{X}$ & 1 & $\mathrm{X}$ & 0 \\
\hline
\end{tabular}

\begin{tabular}{|c|c|c|c|c|c|}
\hline & $\mathrm{A}$ & $\mathrm{B} 1$ & $\mathrm{~B} 2$ & $\mathrm{C}$ & $\mathrm{D}$ \\
\hline $1 \oplus 2$ & $\mathrm{X}$ & $\mathrm{X}$ & $\mathrm{X}$ & 1 & $\mathrm{X}$ \\
$1 \oplus 3$ & $\mathrm{X}$ & $\mathrm{X}$ & 1 & $\mathrm{X}$ & $\mathrm{X}$ \\
$\mathrm{I} \oplus 4$ & $\mathrm{X}$ & $\mathrm{X}$ & $\mathrm{X}$ & 1 & $\mathrm{X}$ \\
\hline & $\mathrm{X}$ & $\mathrm{X}$ & 1 & 1 & $\mathrm{X}$ \\
\hline
\end{tabular}

Tables III. CARE-sets with marked difference vectors, and derived COI(L).

Example 2. Consider the circuit given in Fig.2, and its specification in Fig.1. Fig.3 yields the corresponding ON/OFF AND/OR-trees, and the ON/OFF-sets derived by traversing these trees. In order to get appropriate resolution in this case, with the fanout $B \rightarrow B_{1}, B_{2}$, we distinguish between $B_{1}$ and $B_{2}$ in the vector sets, as shown in Tab. III. We display the original CARE-set with enumerated difference vectors, the corresponding CARE-set as created over the fanout variables $\left(B_{1}, B_{2}\right)$, 
bit-by-bit XOR of each vector in $D_{0}$ with each vector in $D_{1}$, and the resulting $O R$ operation on these vectors, leading to: $\langle\mathrm{XX} 11 \mathrm{X}\rangle$. The " 1 's" indicate the position of the "inputs" in the Cone of Influence for the error location $\mathrm{L}$. Hence, COI(L) = $\left\{B_{2}, C\right\}$, and by inspecting Fig. 2 we easily deduce that $L=F$. By inverting $F(O R \rightarrow$ NOR), we obtain a corrected circuit. A new difference set computation will show that this was indeed the design error. End Example 2.

\section{Exact Diagnosis of Single "Wrong Gate Type" Errors}

The SINGLE_WRONG_GATE_TYPE error hypothesis postulates that by exchanging one gate in the circuit with another gate type, the faulty circuit will be converted to a correct circuit. Tab. IV yields possible gate exchanges.

\begin{tabular}{|c|c|c|c|c|}
\hline $\mathrm{m}_{\mathrm{m} / \mathrm{l}} \mathrm{sec}$ & AND & OR & NAND & NOR \\
\hline AND & & 1 & - & II \\
\hline OR & I & & ॥ & - \\
\hline NAND & - & $\|$ & & 1 \\
\hline NOR & $\|$ & - & I & \\
\hline
\end{tabular}

Table IV. Set of defined gate exchanges.

The entries marked "-" indicate inverting errors, which have been covered in the previous section. The remaining entries are classified as I or II, respectively:

I : monotonic change of gate output,

II: non-monotonic change of gate output.

Class I will be postulated if the change in the circuit function is monotonic. Note that there is exactly one exchange candidate when monotonicity has been established.

We will begin by developing a working algorithm for tree-structured circuits, and use this as a heuristic for non tree-structured circuits also. The algorithm is derived from the following observations, which we for brevity present without proof.

Proposition 1. The ternary ON/OFF input vector sets for a tree-structured circuit derived from the circuit's AND/OR graphs, represent an irredundant set of prime implicants.

Corollary 2. The SINGLE_WRONG_GATE_TYPE error hypothesis with class I errors implies $\mathrm{Y}=\mathrm{X}$ for every vector in the difference set.

Corollary 3. The SINGLE_WRONG_GATE_TYPE error hypothesis with class II errors implies $Y=X$ for every vector in either $D_{0 X}$ or $D_{1 X}$.

For circuits with reconvergent fanouts, the following proposition is valid:

\section{Proposition 2:}

If $D_{0}=E M P T Y$, then $Y<W$, and the wrong gate is of class $I$.

If $D_{1}=E M P T Y$, then $W<Y$, and the wrong gate is of class $I$. 
If Prop. 2 does not hold, then we can not determine the correct gate class a priori, but it is reasonable to try class II first. By utilizing these propositions and corollaries, and Tab. IV, we can find one or more candidate gates for the SINGLE_WRONG_GATE_TYPE error. The erroneous gate will be in this candidate set, but the right one must be determined by computing the difference set for the changed circuit. The strategy to be used is based upon the assumption that when SPEC yields $\mathrm{Y}=\mathrm{X}$ for some ternary vector $\mathrm{V}$, then the design error is found in a path from one input equal to $X$, to the output. The following algorithm can be exploited to find the gate error:

\section{Algorithm FIND_GATE_ERROR}

1. Compute the difference set $\mathrm{D}=\left\{\mathrm{D}_{01}, \mathrm{D}_{0 \mathrm{X}}, \mathrm{D}_{10}, \mathrm{D}_{1 \mathrm{X}}\right\}$.

2. If only $D_{0 X}$ not EMPTY or only $D_{1 X}$ not EMPTY then gate_class $=I$; else gate_class=II.

3. If gate_class $=I$ then $\mathrm{DD}=\mathrm{D}$ else $\mathrm{DD}=\left\{\mathrm{D}_{0 X}, \mathrm{D}_{1 \mathrm{X}}\right\}$. (* Operating difference set $*$ )

4. For every vector $\mathrm{V} \in \mathrm{DD}$ do begin

for every input $V_{i} \in V$ equal $X$ do

begin

$$
\mathrm{G}=\mathrm{GATE} \text { CANDIDATE}(\mathrm{V}, \mathrm{i}, \mathrm{gate} \text { _class); }
$$
end;

Add $\mathrm{G}$ to Candidate_set;

end;

5. Sort Candidate_set;

6. While D not EMPTY do compute $\mathrm{D}$ with next gate $\mathrm{G}$ from Candidate_set;

7. Return G; (* Erroneous gate found *)

\section{End Algorithm FIND_GATE_ERROR;}

The function GATE_CANDIDATE utilizes Corollary (2) when gate_class=I, and Corollary (3) when gate_class=II. Its construction is based upon the following observation (gate_class $=\mathrm{I}$ ): For every vector in $\mathrm{D}, \mathrm{W}=\mathrm{d}$, where $\mathrm{d} \in\{0,1\}$, while $\mathrm{Y}=\mathrm{X}$. This implies that there must be a path from an input $i$ with value $\mathrm{V}_{\mathrm{i}}=\mathrm{X}$ which has been blocked by the erroneous gate. This gate will have $\mathrm{X}$ as one input, but a controlling value on another input, such that the gate output $\in\{0,1\}$. By exchanging this gate with the gate of class I according to Tab. IV (e.g. OR instead of AND), we have a possible error candidate. This will remain a candidate if the circuit now computes to $\mathrm{W}=\mathrm{X}$.

Similarly, for class II errors, we only consider the $\mathrm{D}_{0 \mathrm{X}}$ and $\mathrm{D}_{1 \mathrm{X}}$ difference sets. Thus, the function GATE_CANDIDATE may be sketched as:

\section{Function GATE_CANDIDATE(V,i,gate_class)}

1. Find first gate $\mathrm{G}$ such that there is a path from input $i$ to $\mathrm{G}$ with at least one input to $G$ equal $X$, and the output of $G \in\{0,1\}$.

2. Exchange $G$ with $G N=T a b l e \_I V\left(G, g a t e \_c l a s s\right)$.

3. If $W(V)=X$, return $G$; else return $E M P T Y$;

\section{End Function GATE_CANDIDATE;}


For circuits with reconvergent fanouts, proposition (1) and corollaries 2,3 are not generally valid, but we can use the algorithm with small modifications. We will demonstrate its use on the circuit given in Fig. 6, which is an erroneous implementation of the function given in Fig. 1.

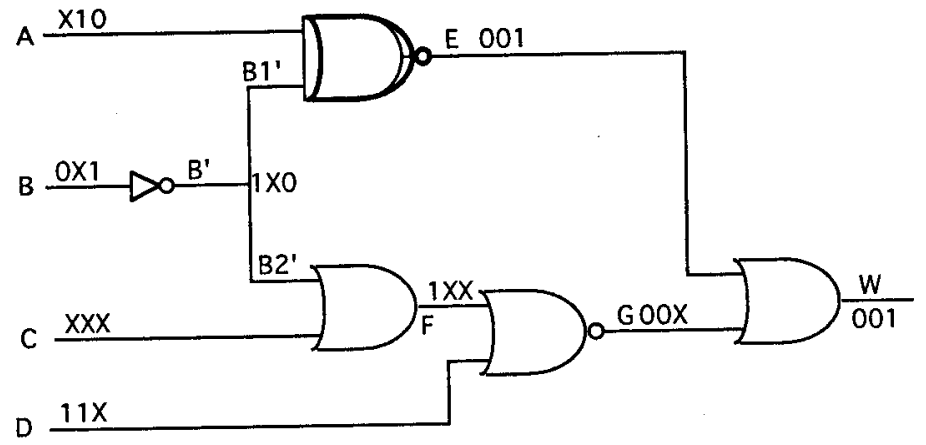

Fig. 6 Erroneous IMPL of Fig.1. Gate E should be AND.

The difference sets are easily found to be (bit order: $\mathrm{ABCD}$ ):

$$
\mathrm{D}_{01}=\mathrm{EMPTY}, \mathrm{D}_{0 \mathrm{X}}=\{\mathrm{X} 0 \mathrm{X} 1,1 \mathrm{XX} 1\}, \mathrm{D}_{10}=\mathrm{EMPTY}, \mathrm{D}_{1 \mathrm{X}}=\{01 \mathrm{XX}\} \text {. }
$$

The three difference vectors are imposed on Fig. 6. The first vector is $A B C D=X 0 X 1$, where we trace $A=X$ to gate $E$. Its output value (0) may be changed to $X$ if we exchange $E$ with AND or NAND, and this change will be propagated to the output W. Thus, $E=\{A N D, N A N D\}$ are gate candidates. We may also trace $C=X$ to gate $F$, and change $F$ to AND or NAND, but this will not result in $W=X$, and hence $F$ is not added to the Candidate_set. Similarly, $A B C D=1 X X 1$ yields $E$ and $G$ as candidate gates, and $\mathrm{ABCD}=01 \mathrm{XX}$ leads to $\mathrm{W}$ as a candidate gate. By recording the number of times a gate is considered to be a candidate, and sorting the candidates accordingly, we normally avoid an extensive search for the correct gate. In this example, we try E first, and find that $\mathrm{E}(\mathrm{NOR} \rightarrow \mathrm{AND})$ yields $\mathrm{D}=\mathrm{EMPTY}$, and hence the error is found.

\section{Conclusion and Further Work}

We have exposed the problem of design error diagnosis, which immediately arises when an implementation IMPL is not proven to be functionally equivalent to a specification SPEC. We have presented a stratagem to diagnose and correct the error, based upon error hypotheses, and likelihood of error occurrence. As an example of the development of suitable algorithms and heuristics, we showed how single inverter errors and wrong gate type errors cculd be diagnosed.

However, there is still much research left on diagnosis before efficient, all round methods can be declared to be in operation. We will focus on developing more heuristics to cover other classes of design errors.

\section{Acknowledgement}

We gratefully acknowledge the kind support of Dominique Borrione at the IMAGARTEMIS Laboratory of Joseph Fourier University, Grenoble, France. 


\section{References.}

[AAS92] E.J. Aas, K.A. Klingsheim and T. Steen, "Quantifying Design Quality: A Model and Design Experiments", Proc. EuroASIC'92, IEEE Comp. Soc., 1992, pp. 172-177.

[BOR92] D.D. Borrione, L.V. Pierre and A.M. Salem, "Formal Verification of VHDL Descriptions in the Prevail Environment", IEEE Design and Test, Vol. 9, No. 2, June 1992, pp. 42-56.

[BRY86] R.E. Bryant, "Graph-Based Algorithms for Boolean Function Manipulation", IEEE Trans. on Comp., Vol. C-35, No. 8, Aug. 1986, pp. 677-691.

[BRY91a] R.E. Bryant, "A Methodology for Hardware Verification Based on Logic Simulation", Journ. of the ACM, Vol. 38, No. 2, April 1991, pp. 299-328.

[BRY91b] R.E. Bryant, D.L. Beatty and C.J.H. Seger, "Formal Hardware Verification by Symbolic Ternary Trajectory Evaluation", Proc.28th DAC, 1991, pp. 397-402.

[CLA91] L.J,M. Claesen, F. Proesmans, E. Verlind and H. De Man, "SFG-Tracing: a Methodology for the Automatic Verification of MOS Transistor Level Implementations from High Level Behavioral Specifications", Proc. ACM/SIGDA Int. Workshop on Formal Methods in VLSI Design, Miami, January 1991.

[GUP92] A. Gupta, "Formal Hardware Verification Methods: A Survey", Journ. of Formal Methods in System Design", Vol. 1, 1992, pp. 151-238,

[HWA85] K.S. Hwang and M.R. Mercer, "Derivation and Refinement of Fanout Constraints to Generate Tests in Combinational Logic Circuits", Proc. ICCAD-85, 1985.

[KUO92] S.Y. Kuo, "Locating Logic Design Errors via Test Generation and Don't Care Propagation", Proc. Euro-DAC, 1992, pp. 466-471.

[MAD88] J.C. Madre and J.P. Billon, Proving Circuit Correctness using Formal Comparison between Expected and Extracted Behaviour", Proc. 25th DAC, 1988.

[MAD89] J.C. Madre, O. Coudert and J.P. Billon, "Automating the Diagnosis and the Rectification of Design Errors with PRIAM", Proc ICCAD, 1989, pp. 30-33.

[SEL68] F.F. Sellers, M.Y. Hsiao and L.W. Bearnson, "Analyzing Errors with the Boolean Difference", IEEE Trans. Comp., Vol. EC-17, July 1968, pp. 676-683.

[TAM89] K.A. Tamura, "Locating Functional Errors in Logic Circuits", Proc. 26th DAC, 1989, pp. 185-191.

[TOM90] M. Tomita and H.H. Jiang, "An Algorithm for Locating Logic Design Errors", Proc ICCAD, 1990, pp. 468-471.

[WEI85] R.S. Wei and A. Sangiovanni-Vincentelli, "PLATYPUS: A PLA Test Pattern Tool", Proc. 22nd DAC, 1985, pp.197-203. 